\title{
Development of Virtual Modelling Techniques in Civil Engineering
}

\author{
Di Wu \\ School of Civil and Environmental Engineering, University of Technology Sydney, Australia \\ di.wu-1@uts.edu.au
}

\begin{abstract}
In modern civil engineering, the design of fit-for-purpose real-world projects is often confronted with many profound difficulties such as variability in ground and climatic conditions, operational requirements, as well as mercurial environmental attacks. These complications aggravate the degradation of physical components of systems and consequently, increasing their probability of failure. One way to solve this issue is to adopt a relatively higher safety factor to enhance their performance against various impacts. However, the effect of selecting a higher safety factor is quite limited or even impossible due to limited funds and resources. An alternative solution is designing a periodic maintenance within a set timeframe, usually its service life. However, challenges that hinder the efficient implementation of an effective maintenance scheme are (i) high costs associated with conservative maintenance scheduling without recognising its current condition and (ii) physical challenges associated with inspection of systems.

While the integrity of civil engineering systems can be reinforced, their sustainability also needs careful attention. One effective approach is to increase service life of the system so the damage to the natural environment can be minimised on a long-term scale. Fortunately our modern technology is capable of delivering high-quality, more-durable systems which exceed their designated service lives. However, a major drawback is the non-recognition of the exact service lives after their designated ones. This dilemma manifests uncontrolled rise in the probability of failure with respect to time that exceeds their designated service lives.

To successfully blend the sustainability factor into the conventional cost-benefit formulation, it is necessary to discover a new engineering practice framework to balance every aspects of the project. Instead of abandoning the established practice framework, researchers should enrich it by adding new components to achieve additional benefits including: (i) transferring periodically scheduled maintenance scheme to a condition-based maintenance scheme; (ii) elongating the service life by controlling the performance of the system; and (iii) improving the service quality by improving the integrity with reduced operational cost. To enrich our current civil engineering practice with new functionalities, the introduction of virtual modelling technique into civil engineering is essential. It will create new opportunities to practise civil engineering in a more effective, controllable, cost-effective, and modular fashion.
\end{abstract}

